\title{
The impact of anxiety on lactose intolerance symptoms*
}

\author{
Veronica Ojetti ${ }^{1 \#}$, Tortora Annalisa ${ }^{1}$, Ianiro Gianluca ${ }^{1}$, Bertucci Flavio ${ }^{1}$, Ferrarese Daniele ${ }^{1}$, \\ Buccelletti Francesco $^{2}$, Zuccala' Giuseppe ${ }^{2}$, Gasbarrini Antonio ${ }^{1}$ \\ ${ }^{1}$ Department of Internal Medicine and Gastroenterology, Catholic University, Rome, Italy \\ ${ }^{2}$ Department of Emergency Medicine, Catholic University, Rome, Italy \\ Email: ${ }^{*}$ veronica.ojetti@tin.it
}

Received 15 February 2013; revised 24 March 2013; accepted 2 May 2013

Copyright (c) 2013 Veronica Ojetti et al. This is an open access article distributed under the Creative Commons Attribution License, which permits unrestricted use, distribution, and reproduction in any medium, provided the original work is properly cited.

\section{ABSTRACT}

BACKGROUND Lactose malabsorption is variably associated with lactose intolerance (LI). The determinants of symptom onset have not yet been completely identified. Visceral sensitivity may play a role in the pathogenesis of functional symptoms; an increasing bulk of clinical and research data suggests the importance of the brain-gut interaction in intestinal bowel syndrome. AIM: To assess the impact of anxiety on LI symptoms, and the correlation between gas production during Lactose Breath test (LBT) and the intensity of gastrointestinal symptoms (GI) in anxious (A) and non-anxious (NA) patients. METHODS: LBT was carried out in 104 consecutive participants referred to our Gastroenterology Unit. GI symptoms were evaluated using the visual analogue scale (VAS: 0-10) throughout the LBT. All patients completed the HADS to determine anxiety. RESULTS LBT was positive in 70/104 (67\%) participants; 49/ 104 (47\%) showed significant anxiety symptoms (>10 items). In A subjects we did not find any significant correlation between gas levels and abdominal LI symptoms scores throughout the LBT. A subject showed higher scores for all LI symptoms as compared with other participants, independently of LBT results. NA pts with positive LBT showed higher scores for all LI symptoms, as compared to negative subjects. LIMITATIONS: The predominant gender is female but it is in agreement with literature data that show a preponderance of IBS in women. CONCLUSIONS: A status significantly influences the severity of all LI symptoms and does not strictly correlate with lactase deficiency. Conversely, among NA subjects LI symptoms increase along with $\mathrm{H}_{2}$ production. Anxiety seems to be a confounding factor in IBS pts.

\footnotetext{
*The authors declare that they have no conflict of interest.

"Corresponding author.
}

Keywords: Lactose Intolerance; Anxiety; $\mathrm{H}_{2}$ Breath Test

\section{INTRODUCTION}

Lactose intolerance (LI) is a common condition affecting a large proportion of the world population [1]; its prevalence varies among countries [2]. In Europe, the prevalence increases towards the South and East, reaching $70 \%$ in southern Italy [3].

Lactose malabsorption is a common condition characterized by a deficiency of lactase, which is an enzyme of the brush border membrane of the intestinal mucosa that hydrolyzes lactose into galactose and glucose. The uncleaved lactose passes intact into the colon; the enteric bacteria quickly ferment it and produce large amounts of gas $\left(\mathrm{CO}_{2}, \mathrm{H}_{2}, \mathrm{CH}_{4}\right)$ that are absorbed and then excreted in breath or stools. About $14 \%-20 \%$ of $\mathrm{H}_{2}$ released in the colon is excreted by the lungs [4]; so, the measurement of $\mathrm{H}_{2}$-breath concentration reflects intestinal production [5].

In addition, like with other unabsorbed sugars, lactose and its fermentation products raise the osmotic pressure of the colon contents, which causes symptoms like diarrhea.

The presence of unabsorbed lactose in the gut lumen does not necessarily determine gastrointestinal symptoms. Only when lactose malabsorption is clinically manifest with symptoms like flatulence, bloating, diarrhea, abdominal pain, lactose intolerance does occur. Symptoms range from mild to severe, varying in relation to the amount of lactose consumed and the visceral sensitivity $[6,7]$. Recently, psychosocial stress has also been implicated in the degree of symptoms. The relationship between the gastrointestinal tract and the immune system is called "brain-gut axis" (BGA), and is particularly influenced by different stressors. In recent years, the important interplay between stress and gut has been evidenced and studied. Many gastrointestinal functions such as gut 
motility, gastric secretion, mucosal permeability and barrier function and also visceral sensitivity may be affected by stress. The same gut microbiota can change and respond directly to stress-related host signals. It is well known that exposure to stress may lead to the onset of different gastrointestinal symptoms such as dyspepsia, diarrhea or abdominal pain. Dysregulation of BGA induced by stress may lead to the development of a broad array of gastrointestinal diseases, including gastro-esophageal reflux disease, peptic ulcer disease, inflammatory bowel disease, irritable bowel disease and even food allergy [8].

Community studies of food allergies and sensitivities have shown a large discrepancy between the prevalence of perceived food intolerance and of real food intolerance diagnosed by objective methods. Many papers reveal that subjects with food intolerance or allergy have a poor quality of life, and the psychological setting influences the development and severity of symptoms. It has been suggested the misperception of food intolerance arises largely from psychiatric illness or personality disorders. Then, the interplay between gut and mind is often difficult to evaluate [9-11].

Aim of our study was to assess the correlation, if any, between gas production during the lactose breath test (LBT) and the intensity of gastrointestinal symptoms in anxious (A) and non-anxious (NA) patients, as well as the impact of anxiety on LI symptoms.

\section{METHODS}

This prospective observational study was performed in the Gastroenterology and Internal Medicine outpatient Unit of the Gemelli University Hospital, Rome. Informed consent was obtained from all the participants.

We evaluated 104 (19 M/85 F, mean age $44.5 \pm 16$ yrs) consecutive patients referring to our Gastroenterology Unit from September to December 2010 for gastrointestinal symptoms following lactose ingestion, who performed a $\mathrm{H}_{2} / \mathrm{CH}_{4} \mathrm{LBT}$.

The study was performed in accordance with the Declaration of Helsinki, and approved by the local Ethics Committee.

\subsection{Lactose Breath Test}

To minimize basal hydrogen excretion, patients were asked to have a carbohydrate-restricted dinner on the day before the test and to be fasting for at least $12 \mathrm{~h}$ on the testing day. Before starting the test patients performed a mouth wash with $20 \mathrm{ml}$ chlorhexidine $0.05 \%$. Smoking and physical exercise were not allowed for $30 \mathrm{~min}$ before and during the test. Breath samples were collected immediately before lactose ingestion. Then a dose of $25 \mathrm{~g}$ of lactose was administered and breath samples were taken every $30 \mathrm{~min}$ for $4 \mathrm{~h}$ and analyzed immediately for $\mathrm{CH}_{4} / \mathrm{H}_{2}$ using a Breath Tracker Quintron Gas Chromatograph (Quintron Instrument Company, Milwaukee, WI, USA). Results were expressed as parts per million (p.p.m.). LBT was considered positive for lactose malabsorption when an increase in $\mathrm{H}_{2}$ value exceeding 20 parts per million (p.p.m.) over the baseline value was registered [12].

\subsection{Symptoms Assessment and Study Outcomes}

For a total of $4 \mathrm{~h}$ (during the LBT), at each blow for a total of nine times all patients were invited to fulfill a diary where they recorded the eventual occurrence of intolerance symptoms (bloating, abdominal pain, flatulence and diarrhea), which severity was assessed by a visual analogical scale (the score ranging from 0 , absent to 10 , severe).

Before the LBT test each patient also completed the anxiety and depression scale (HADS).

The primary outcomes were the association of anxiety with the development of LI symptoms during a LBT and the possible correlation between gas production and the intensity of LI symptoms during a LBT in A and NA pts.

\subsection{Statistical Analysis}

Statistical analysis was carried out with STATA 11 (Texas, USA). All variables were normally distributed and variables concerning $\mathrm{H}_{2}$ excretion and clinical score were expressed as mean values $\pm \mathrm{SD}$. Correlations were assessed using the Spearman RHO test. Calculated pvalues less than 0.05 were considered statistically significant.

\section{RESULTS}

We enrolled in the study 104 patients, whose demographic characteristics are shown in Table 1. Of these participants, 51 (49\%) were classified as anxious (A), as they scored $>10$ items in the HADS test, while 53 (51\%) resulted non-anxious (NA), with $<7$ items. Also, 70 participants $(67 \%)$ resulted positive to LBT; of these, 40 belonged to A group, and 30 to NA group.

NA patients with a positive LBT revealed significantly higher scores for all LI symptoms, as compared to nega-

Table 1. The demographic characteristic of subject analysed divided into anxious and non anxious patients.

\begin{tabular}{ccc}
\hline & Anxious & Non anxious \\
\hline Total no pts (\%) & $51(49 \%)$ & $53(51 \%)$ \\
Sex no (M/F) & $5 / 46$ & $14 / 39$ \\
Mean age (yrs) & $43.5 \pm 18$ & $46.7 \pm 14$ \\
LBT pos no (\%) & $40(43.4 \%)$ & $30(56.6 \%)$ \\
\hline
\end{tabular}

LBT: lactose breath test. 
tive ones: bloating $2.2 \pm 1.7$ vs $1.6 \pm 2$ p $<0.05$, abdominal pain $0.9 \pm 1.3$ vs $0.9 \pm 1.8 \mathrm{p}=0.98$, flatulence $1.6 \pm 2.2$ vs $1 \pm 1.9 \mathrm{p}<0.05$ diarrhoea $0 \pm 0$ vs $0 \pm 0.25$ $\mathrm{p}<0.01$, respectively (Figure 1). In the latter subset of pts we found a significant association of $\mathrm{H}_{2}$ concentration with bloating $(\mathrm{R}=0.31,95 \% \mathrm{CI} 0.04-0.54 ; \mathrm{p}=$ $0.02)$, abdominal pain $(\mathrm{R}=0.40,95 \% \mathrm{CI} 0.14-0.61 ; \mathrm{p}=$ $0.003)$ and flatulence $(\mathrm{R}=0.37,95 \% \mathrm{CI} 0.10-0.58 ; \mathrm{p}=$ 0.007).

Conversely, among A pts we did not find any significant association between abdominal LI symptoms and lactose breath test results: bloating $2.8 \pm 5.9$ vs $2.4 \pm 2.7$ $\mathrm{p}=0.7$, abdominal pain $1.5 \pm 2.3$ vs $1.7 \pm 2.3 \mathrm{p}=0.28$, flatulence $1.6 \pm 1.6$ vs $1.8 \pm 2.3 \mathrm{p}=0.35$, diarrhoea $0.4 \pm$ 1.1 vs $0.1 \pm 0.8 p=0.43$ (Figure 1). Moreover, we did not find any correlation between gas levels and abdominal LI symptom scores throughout LBT. Interestingly, A pts with negative lactose breath test showed mean scores for all LI symptoms higher than those of NA pts.

In these participants the $\mathrm{CH}_{4}$ production was not associated with any of the symptom scores analyzed.

\section{DISCUSSION}

In our study we evaluated the real impact of anxiety on LI symptoms during a LBT.

We showed that anxious status much influence the severity of all LI symptoms and the presence of LI symptoms in A pts do not strictly correlate with lactase defi- ciency. Moreover in this subset of pts LI symptoms did not correlate with gas production neither $\mathrm{H}_{2}$ or $\mathrm{CH}_{4}$. It is well-known that psychological factors, can enhance pain perception and trigger pain facilitation processes.

Previous studies confirm that irritable bowel syndrome (IBS) patients have more psychological symptoms than healthy controls, and these symptoms are associated with increased pain perception [13].

Addolorato et al. [14] confirmed a possible association via anxiety and depression between IBS and food intolerance. They hypotized that anxiety and depression in IBS and food intolerance patients could be responsible for an increase in the activity of the autonomic nervous system and could provoke motility alterations through mast cell mediator release as well as enhancing release due to allergen cross linking with IgE at the mast cell surface.

Meanwhile, in NA subject, we showed that LI symptoms increase according to the $\mathrm{H}_{2}$ production and were strictly associated with a positive LBT.

It has been assumed that luminal distension via a combination of osmotic effects and gas production related to their rapid fermentation by bacteria in the intestine trigger gastrointestinal symptoms.

In this subset of pts self-reported milk-intolerance symptoms could predict a positive LBT.

In particular adjusting for the anxiety level diarrhea seems to be the most significant symptoms related to LBT results.

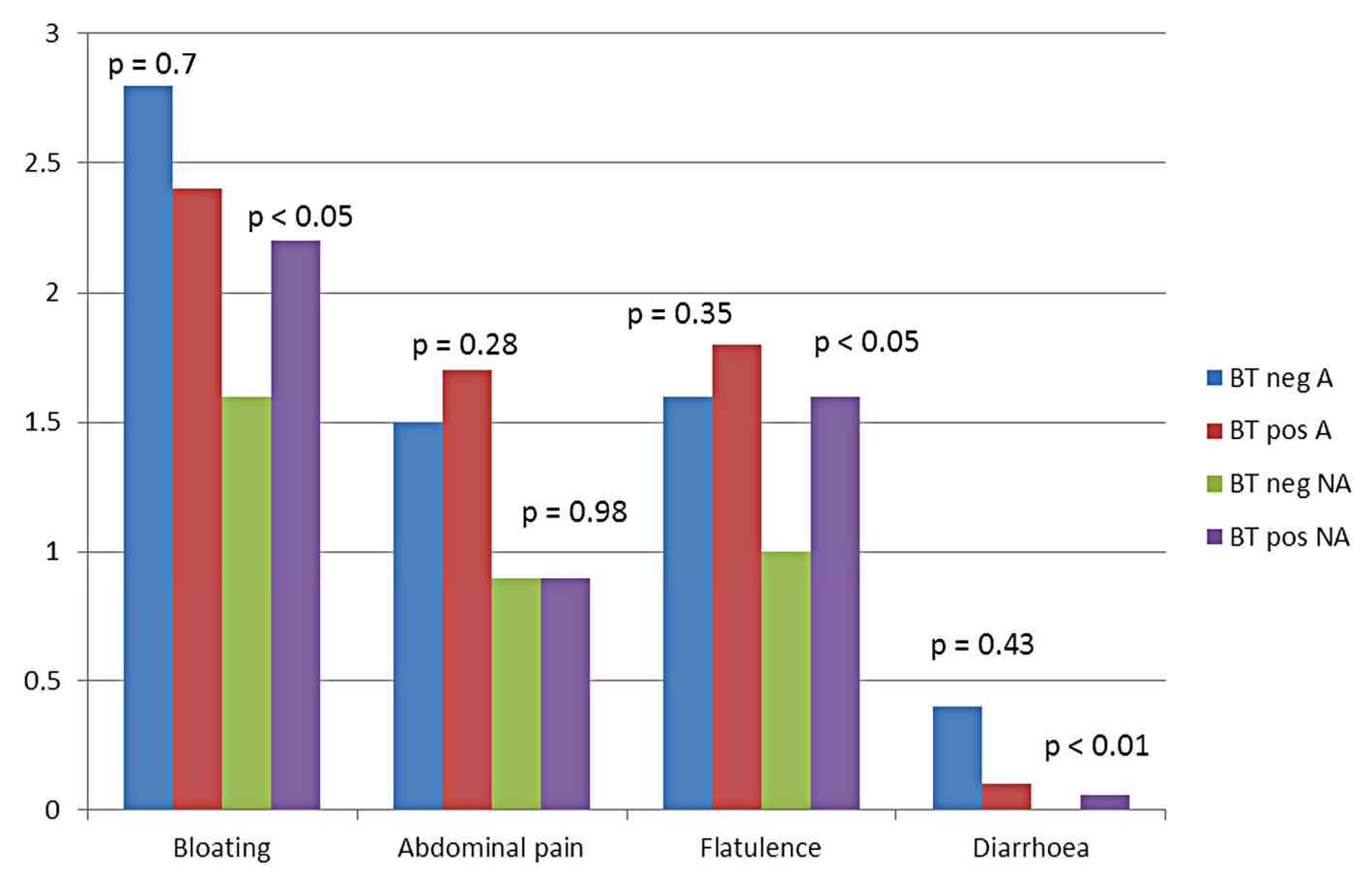

BT: breath test; Neg: negative; Pos: positive; A: anxius; NA: non anxious.

Figure 1. Comparison of lactose intolerance symptoms in patients with positive (BT pos) and negative (BT neg) lactose breath test according to the anxious status (A vs NA). 
Our results agrees with a recent study who demonstrates that bloating and diarrhea are the most frequently reported symptoms in lactose intolerant pts and the amount of exhaled hydrogen is positively correlated with the severity of the gastrointestinal symptoms [15].

Moreover our study demonstrates that the $\mathrm{CH}_{4}$ producers fail to correlate with any of the Symptoms' scores analyzed. These observations imply that hydrogen produced during lactose fermentation will occupy a relatively greater space in the intestinal lumen respect to methane, since four liters of hydrogen are used to produce one liter of methane [16].

A limitation of our study is that there are more female than male, but this is in agreement with literature data on the high preponderance of IBS in women.

In conclusion, according to our opinion, anxiety seems to be a confounding factor in IBS pts

And the presence of LI symptoms, even if severe, in A pts does not correlate with a positive LBT.

If these results would confirmed by other studies, we suggest to evaluate in IBS pts with food intolerance symptoms, consider the degree of anxiety to better focus this subset of pts and prescribe a more specific treatment based also on a psychological approach since psychosomatic disorders should be identified early in order to avoid long lasting and frustrating investigations.

\section{REFERENCES}

[1] Swagerty, D.L., Walling, A.D. and Klein, R.M. (2002) Lactose intolerance. American Family Physician, 65, 1845-1850.

[2] Sahi, T. (1994) Genetics and epidemiology of adult type hypolactasia. Scandinavian Journal of Gastroenterology, 29, 7-20. doi:10.3109/00365529409091740

[3] Vuorisalo, T., Arjamaa, O., Vasemagi, A., Taavitsainem, J.P. and Tourunen, A. (2012) Saloniemi. Perspectives in Biology and Medicine, 55, 163-174. doi:10.1353/pbm.2012.0016

[4] Levitt, M.D. (1969) Production and excretion of hydrogen gas in man. New England Journal of Medicine, 281, 122-127. doi:10.1056/NEJM196907172810303

[5] Levitt, M.D. and Donaldson, R.M. (1970) Use of respiratory hydrogen (H2) excretion to detect carbohydrate malabsorption. Journal of Laboratory and Clinical Medicine, 75, 937-945.
[6] Gudmand-Hoyer, E. (1994) The clinical significance of disaccharide maldigestion. American Journal of Clinical Nutrition, 59, 735S-741S.

[7] Calrk, G., Quigley, E.M., Cryan, J.F. and Dinan, T.G. (2009) Irritable bowel syndrome: Towards biomarker identification. Trends in Molecular Medicine, 15, 478-489. doi:10.1016/j.molmed.2009.08.001

[8] Konturek, P.C., Brzozowski, T. and Konturek, S.J. (2011) Stress and the gut: Pathophysiology, clinical consequences, diagnostic approach and treatment options. Journal of Physiology and Pharmacology, 62, 591-599.

[9] Tosic-Golubovic, S., Miljkovic, S., Nagorni, A., Lazarevic, D. and Nikolic, G. (2010) Irritable bowel syndrome, anxiety, depression and personality characteristics. Psychiatria Danubina, 22, 418-424.

[10] Cho, H.S., Park, J.M., Lim, C.H., Cho, Y.K., Lee, I.S., Kim, S.W., Choi, M.G., Chung, I.S. and Chung, Y.K. (2011) Anxiety, depression and quality of life in patients with irritable bowel syndrome. Gut and Liver, 5, 29-36.

[11] Jones, M., Koloski, N., Boyce, P. and Talley, N.J. (2011) Pathways connecting cognitive behavioral therapy and change in bowel symptoms of IBS. Journal of Psychosomatic Research, 70, 278-285.

[12] Strocchi, A., Corazza, G.R., Anania, C., Benati, G., Malservisi, S., Cherchi, M.V., et al. (1997) Quality control study of H2 breath testing for the diagnosis of carbohydrate malabsorption in Italy. The "Tenue Club” Group. Italian Journal of Gastroenterology and Hepatology, 29, 122-127.

[13] Hartono, J.L., Mahadeva, S. and Goh, K.-L. (2012) Anxiety and depression in various functional gastrointestinal disorders: Do differences exist? Journal of Digestive Diseases, 13, 252-257. doi:10.1111/j.1751-2980.2012.00581.x

[14] Addolorato, G., Marsigli, L., Capristo, E., Caputo, F., Dall'Aglio, C. and Baudanza, P. (1998) Anxiety and depression: A common feature of health care seeking patients with irritable bowel syndrome and food allergy. Hepatogastroenterology, 45, 1559-1564.

[15] Erminia, R., Ilaria, B., Tiziana, M., Silvia, P., Antonio, N., Pierpaolo, D. and Loris, B. (2011) HRQoL questionnaire evaluation in lactose intolerant patients with adverse reactions to foods. Internal and Emergency Medicine.

[16] Gasbarrini, A., et al. (2009) Methodology and indications of H2-breath testing in gastrointestinal diseases: The Rome Consensus Conference. Alimentary Pharmacology \& Therapeutics, 29, 1-49. 